\title{
Activated events in glasses: the structure of entropic droplets
}

\author{
Maxim Dzero $^{1}$, Jörg Schmalian ${ }^{1}$ and Peter G. Wolynes ${ }^{2}$ \\ ${ }^{1}$ Department of Physics and Astronomy and Ames Laboratory, Iowa State University, Ames, IA 50011 and \\ ${ }^{2}$ Department of Chemistry and Biochemistry and Department of Physics, \\ University of California, San Diego, La Jolla, CA 92093
}

(Dated: September 12, 2021)

\begin{abstract}
Using an effective potential approach, we present a replica instanton theory for the dynamics of entropic droplets in glassy systems. Replica symmetry breaking in the droplet interface leads to a length scale dependent reduction of the droplet surface tension and changes the character of the dynamical heterogeneity and activated dynamics in glasses.

PACS numbers: 64.70Pf, $75.10 \mathrm{Nr}, 05.70 \mathrm{Ln}$
\end{abstract}

At it's most basic level the puzzle presented by the existence of glasses is "how can any disordered arrangement of atoms be stable?". Disorder implies myriads of structural possibilities - why don't the unguided thermal motions of the atoms in a glass take advantage of these possibilities and lead to ceaseless change and flow? Actually, glasses do flow, but only very slowly. Thus our naïve question must take on a more quantitative form"why do materials with apparently high entropy density flow as slowly as they do?" Molecules in ordinary glass undergo displacements greater than an angstrom only on time scales greater than hours. Answering this quantitative question is tantamount to a theory of the glass transition.

The most successful treatment of this issue to date is provided by the random first order transition (RFOT) theory of supercooled liquids and glasses 1, 2, 3, 4, 5, 6, 7]. Mean field calculations on these systems suggest a discontinuous transition without latent heat at a Kauzmann temperature $T_{K}$, as found in infinite range spin glasses that lack reflection symmetry [8], which is called "random first order". The mean field approaches include the use of replicas [2, 4, 5, 6, 7, 8, 9] and dynamical theories [10, 11. and exhibit 1-step replica symmetry breaking at $T_{K}$ and a dynamical crossover temperature, $T_{A}>T_{K}$, for the onset of activated dynamics. Although a system with infinite range forces can break ergodicity while retaining a macroscopic configurational entropy, this is impossible for a system with finite range interactions 2, 12. Ergodicity will be restored by "entropic droplets", in which a region of the frozen solid is replaced by any of an exponentially large number of alternatives. The entropy due to these many alternatives acts as a driving force for structure change which is, however, off-set by the free energy cost of matching two alternative structures at their boundaries. This conflict gives the free energy barrier for slow activated motions.

Many quantitative aspects of the glassy dynamics of molecular liquids have been predicted using the RFOT theory and are found to be in good agreement with experiment. The predictions include the relation between both the mean activation barriers in the liquid and glass regimes 13 and the non-exponentiality of relaxation 14 with the configurational heat capacity, the size scale of the mosaic structures 13, which show up as "dynamical heterogeneities" in the laboratory [15], as well as a quantitative prediction of the anomalous low temperature properties of glasses, including their linear specific heat [16]. These successes of the RFOT theory encourage us to put the approximations presently used on firmer formal ground. This will allow the extension of the RFOT theory to systems more exotic than structural glasses such as colloidal systems having stripes or lamellae, i.e. micro-emulsions, which are predicted to be in the same universality class 17, or to glassy phases with strongly quantum degrees of freedom such as the electronic motions of correlated electron systems [18].

In this paper, using replica based methods, we study droplet dynamics in a frozen glassy system and show how it differs fundamentally from nucleation in ordinary first order transitions. These calculations yield a temperature and length scale dependent reduction of the droplet surface tension that strongly affects the dynamical heterogeneity and activated dynamics of glassy systems. Specifically, we show that the transition states in a statistical field theory are instantons possesing additional replica symmetry breaking (RSB) in the entropic droplet and argue that this new RSB state arises from configurational entropy fluctuations. Instanton like theories that ignore this complexity, particularly at the droplet interface have been presented earlier 2, 19, 20, 21]. Our results demonstrate that those approaches contain instabilities while the present RSB solution strongly supports the wetting picture put forward in $[\underline{3}$.

We consider a system of interacting particles that becomes glassy at low temperatures. Recent progress in the replica description of glass-forming systems with entropy crisis [4, 5, 6] allows us to formulate the RFOT theory in terms of a spatially varying collective variable, $q_{a b}(r)$, that corresponds to the local Debye Waller factor of molecular motions for liquid systems. $q_{a b}=0$ in the ergodic liquid while $q_{a b} \rightarrow O(1)$ for $a \neq b$ in case of perfect long time correlations. Within replica mean field theory $[4,[5,[6]$ the long time density corre- 
lations approach $q \rho_{0} S(k)$, with the equilibrium liquid structure factor being $S(k)$, the mean particle density $\rho_{0}$ and the off-diagonal element $q$ of $q_{a b}$. Below the dynamic transition temperature $T_{A}, q$ becomes nonzero for the first time and an extensive configurational entropy $S_{c}$ occurs, that vanishes linearly at the Kauzmann temperature $T_{K}<T_{A}$. A heterogeneity of the glass, i.e. the emergence of correlated long time dynamics over certain length scales, is reflected in an $r$-dependence of $q_{a b}(r)$.

The mean field value and fluctuations of $q_{a b}$ are determined by an effective Hamiltonian, $H[q]$. In order to keep our calculation transparent we will not specifically determine $H[q]$ from an explicit calculation for supercooled liquids (for an example see Ref. [7] ), but start from a simple polynomial model in the same universality class [8]:

$$
H=\sum_{a, b} \int d^{d} r\left(h\left[q_{a b}\right]-\frac{1}{3} \sum_{c} q_{a b} q_{b c} q_{c a}\right)
$$

with $h\left[q_{a b}\right]=\frac{1}{2}\left(\nabla q_{a b}\right)^{2}+\frac{t}{2} q_{a b}^{2}-\frac{1+w}{3} q_{a b}^{3}+\frac{y}{4} q_{a b}^{4}$. In Eq⿴length scales are measured in units of the interparticle distance and energy scales in units of a scale that determines the absolute value of the configurational entropy. The parameters $t, w>0$ and $y>0$, which are in principle all temperature dependent, become dimensionless. We take the primary $T$-dependence to reside in the quadratic term, $t=\frac{T-T_{0}}{T_{0}}$ where $T_{0}$ determines the temperature scale where slow dynamics of the model sets in. We use both the absolute and reduced temperature variables, $T$ and $t$, i.e. $T_{K}$ or $t_{K}=t\left(T_{K}\right)$ etc.

Our aim is to perform an instanton calculation to describe the escape from a given metastable frozen solid configuration. This requires a technique that agrees in the homogeneous limit with other mean field calculations within the RFOT universality class $[\mathbf{5}, 18$, but allows us to study the behavior for arbitrary values of the overlap between two states. This is achieved by the quenched effective potential approach of Franz and Parisi $[\underline{6}$. One considers a fixed overlap $p_{c}(r)=\rho_{1}(r) \rho_{2}(r)$ between two particle density configurations and determines the constrained partition function $Z\left(p_{c}, \rho_{2}\right)=\left\langle\delta\left(p_{c}(r)-\rho_{1}(r) \rho_{2}(r)\right)\right\rangle_{\rho_{1}}$ by thermally averaging over one configuration. The effective potential, $\Omega\left[p_{c}\right]=-T\left\langle\log Z\left(p_{c}, \rho_{2}\right)\right\rangle_{\rho_{2}}$, is the average of the free energy over the second configuration $[\underline{6}]$ and can be written as:

$$
\Omega\left[p_{c}\right]=2 \int d^{d} r h\left[p_{c}\right]+F\left[p_{c}\right] .
$$

Here $F\left[p_{c}\right]=-\left.T \frac{\partial}{\partial m} \int D q \exp \left(-\beta H_{p_{c}}[q]\right)\right|_{m \rightarrow 0}$ is the quenched free energy average for a system with Hamiltonian, Eq in an external field $p_{c}^{2}(r): H_{p_{c}}[q]=H[q]-$ $\sum_{a b} \int d^{d} r p_{c}^{2}(r) q_{a b}(r)$. Replica theory gives the statistical interpretation of $p_{c}$ and $q_{a b} . p_{c}$ refers to the similarity

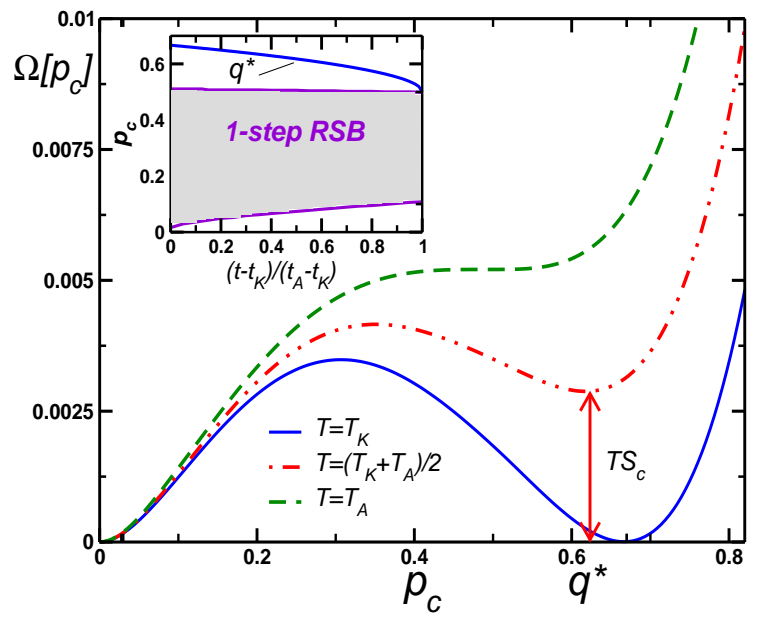

FIG. 1: Effective potential as a function of the overlap, $p_{c}$ for different temperature; inset: region of $p_{c}$ values where RSB occurs together with $q^{*}(t)$.

with the initial state while $q_{a b}$ measures the configurational similarity between different states, regardless of the initial state.

Before we perform the instanton calculation we summarize the homogeneous mean field theory of Eq In Fig.1 we show $\Omega\left[p_{c}\right]$ for $r$-independent $p_{c}$ where $q_{a b}$ in $F\left[p_{c}\right]$ is determined via saddle point method. The minima of $\Omega\left[p_{c}\right]$ determine the mean field value of the Debye Waller factor $q^{*}=\frac{w+\sqrt{w^{2}-4 t}}{2 y}$ and the configurational entropy $\Omega\left[q^{*}\right]=T S_{c}[\underline{\underline{6}}]$. We find $t_{A}=\frac{w^{2}}{4 y}$ and $t_{K}=\frac{2 w^{2}}{9 y}$ for the reduced dynamic crossover and Kauzmann temperatures, respectively. The configurational entropy density, $s_{c}=S_{c} / V$, vanishes linearly at the Kauzmann temperature, $s_{c}\left(t \simeq t_{K}\right)=\frac{1}{4 T_{K}} q^{* 2}\left(t_{K}\right)\left(t-t_{K}\right)$, while $s_{c}\left(t_{A}\right)=\frac{1}{12 T_{A}} q^{* 2}\left(t_{A}\right) t_{A}$. For $p_{c}=q^{*}, q_{a b}$ is replica symmetric. The lowest replicon eigenvalue, $\lambda$, is positive for $t_{K} \leq t<t_{A}$ and vanishes at $t_{A}$ like $\lambda \propto\left(t_{A}-t\right)^{1 / 2}$. Since replica symmetry in the effective potential formalism corresponds to one step RSB in the conventional replica approach 22], all these results are in agreement with the mean field theory of a RFOT. For $p_{c}$ values between 0 and $q^{*}$ an additional replica symmetry breaking in $q_{a b}$ occurs [9, 23] (see inset of Fig.1).

The $\Omega\left(p_{c}\right)$ of Fig.1 clarifies the fact that between $T_{K}$ and $T_{A}$ the frozen solid with $p_{c}=q^{*}$ is metastable with respect to the liquid $\left(p_{c}=0\right)$ and higher in free energy by $T S_{c}$. The system will try to reduce its energy by realizing this entropy via droplet nucleation. We argue that the transition state for this nucleation is determined by an instanton solution for the overlap profile $p_{c}(r)$, i.e. via $\frac{\delta \Omega\left[p_{c}\right]}{\delta p_{c}(r)}=0$ with barrier $\Omega\left(p_{c}\right)-\Omega\left(q^{*}\right)$; see also Ref. 21]

Assuming for the moment replica symmetry for the instanton, $q_{a b}(r)=\left(1-\delta_{a b}\right) q(r)$ we find the solution $p_{c}(r)=q(r)$, determined by $\Delta p_{c}(r)=\frac{d V\left(p_{c}\right)}{d p_{c}(r)}$ with $V\left(p_{c}\right)=\frac{t}{2} p_{c}^{2}-\frac{w}{3} p_{c}^{3}+\frac{y}{4} p_{c}^{4}$. While $V\left(p_{c}\right)$ and $\Omega\left(p_{c}\right)$ dif- 
fer in detail, they have rather similar shape and agree for $p_{c}=q^{*}$. If replica symmetry holds, the problem then becomes almost identical to ordinary homogeneous nucleation [24] with transition temperature $T_{K}$ and spinodal $T_{A}$. Close to $T_{K}$ a thin interface and a diverging droplet size result. The competition between surface and bulk terms determines the barrier

$$
\Delta F=4 \pi \sigma_{0} R^{2}-\frac{4 \pi}{3} T s_{c} R^{3} .
$$

The sole distinction from ordinary nucleation is that the driving force for the decay is entropic. For the surface tension, the model Eq 1yields $\sigma_{0} \simeq q^{* 2}\left(t_{K}\right) t_{K}^{1 / 2}$, the interface width is of order $l_{0}=2^{7 / 2} t_{K}^{-1 / 2}$ and the droplet radius is $R \simeq l_{0} \frac{t_{K}}{t-t_{K}}$. The time scale for activated dynamics therefore diverges like $\log \frac{\tau}{\tau_{0}} \propto\left(\frac{t_{K}}{t-t_{K}}\right)^{d-1}$ at $T_{K}$ for spatial dimension $d>1$. This result was first found in Ref. 2] and also given in 19, 21. Close to $T_{A}$ no sharp droplet interface can be defined [25]. A dimensional analysis of the instanton equation near $T_{A}$ yields the length $\xi_{A} \simeq\left(t_{A}\left(t_{A}-t\right)\right)^{-1 / 4}$, identical to the length scale in mode coupling theory above the upper critical dimension $d_{u c}=6$ [26], see also Ref. 2]. Recently, using the effective potential approach, all these results for replica symmetric $q_{a b}$ were independently obtained by Franz 21].

The self-generated randomness of a glassy system however should modify the nucleation dynamics. A crucial question is whether the replica symmetry for $q_{a b}$ is indeed stable for the droplet. A stability analysis shows that the replicon eigenvalues, $\lambda$, of the droplet solution are determined by

$$
\left(-\nabla^{2}+U(r)\right) \psi(r)=\lambda \psi(r),
$$

with $r$-dependent potential $U(r)=\left.\frac{d^{2} V(q)}{d q^{2}}\right|_{q=q^{*}}$. Without the gradient term we would recover the result for $\lambda$ discussed above and replica symmetry is stable below $T_{A}$. The stability changes if we include, however, the gradient term. Plainly, the replicon eigenvalues are identical to the eigenvalues of the usual fluctuation determinant in the decay of a metastable state. Thus, there is one negative eigenvalue for $T_{K}<T<T_{A}$. Close to $T_{K}$ it follows that $\lambda \simeq-R^{-2}$ with droplet radius $R$. The unstable eigenvector is in the replicon direction and this instability occurs in addition to the expected instability with respect to the growth mode, i.e. the motion of the interface of the droplet. We further infer that the replicon eigenfunction, $\psi(r)$, is localized on the droplet interface.

The instability of the replica symmetric solution entails an additional replica symmetry breaking as the overlap $q_{c}$ goes "over the hill", i.e. close to the interface. Allowing more general solutions for $q_{a b}(r) \rightarrow q(r, x)$, where $x \in[0,1]$ is the usual RSB parameter, we obtain from $\frac{\delta \Omega\left[p_{c}\right]}{\delta p_{c}(r)}=0$ and the saddle point equation for $q_{a b}$, two coupled instanton equations:

$$
\nabla^{2} p_{c}(r)=\frac{d V\left(p_{c}\right)}{d p_{c}(x)}-p_{c}^{2}(r)+p_{c}(r) \int_{0}^{1} q(r, x) d x
$$

and

$$
\begin{aligned}
\nabla^{2} q(r, x) & =\frac{d V(q)}{d q(r, x)}-(1-x) q^{2}(r, x)-p_{c}^{2}(r) \\
& +\int_{0}^{x} d x^{\prime} q^{2}\left(r, x^{\prime}\right)+2 q(r, x) \int_{x}^{1} q\left(r, x^{\prime}\right) .
\end{aligned}
$$

We have numerically solved these equations under the assumption of spherical symmetry and with the approximation that $\nabla^{2} \simeq \frac{d^{2}}{d r^{2}}$, justified for large droplet radius, i.e. for $T$ close to $T_{K}$. We find that new solutions with one step replica symmetry breaking exist! In Fig.2 we show our results for $p_{c}(r)$ and for $q(r, x)$ below $\left(q_{0}(r)\right)$ and above $\left(q_{1}(r)\right)$ the breakpoint, $\bar{x}$. We find $\bar{x}$ to be $r$ - independent. Outside the droplet, $p_{c}$ and the induced density correlations $q(r, x)$ all approach the mean field value $q^{*}$. However, inside of it, $q(r, x)$ has a nontrivial distribution function. With weight $\bar{x}, q(r, x)$ is small $\left(q_{0} \lesssim p_{c}\right)$ and the transition state structures are configurationally distinct. However, with smaller weight, $1-\bar{x}$, a few energetically well matching states contribute to making the transition. This demonstrates that dynamical heterogeneity in glasses differs fundamentally from ordinary nucleation. This instability originates in the interface, where the gradients are nonzero, but affects, via boundary conditions, the overlap distribution in the entire droplet. As $T \rightarrow T_{K}$ we find $\bar{x} \rightarrow 1$. At the Kauzmann temperature, where the replicon eigenvalue vanishes, the system is marginal in a new, heterogeneous sense.

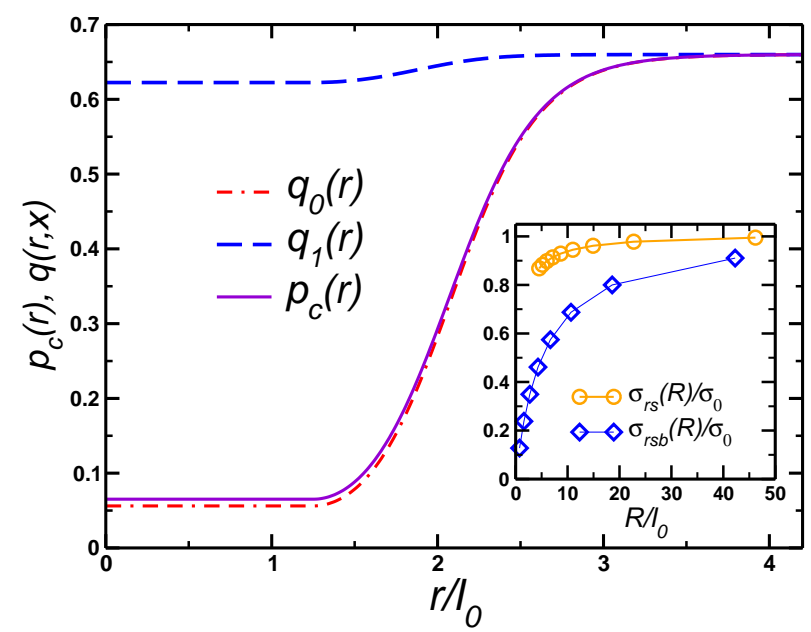

FIG. 2: Instanton profile for $t=t_{K}+0.08\left(t_{A}-t_{K}\right)$ and $y=w=1$ with breakpoint $\bar{x}=0.975$ and $q_{0,1}=q(x \lessgtr \bar{x}, r)$. Inset: surface tension as function of the droplet radius for the RS and RSB instantons.

If we insert our solution into $\Omega\left[p_{c}\right]$ we obtain the interesting result that, compared to the replica symmetric 
case, the bulk driving force for nucleation is unchanged. The new solution does however change the value of the surface tension. Close to $T_{K}$ our numerical results are very well described by $\sigma(t)=\sigma_{0}\left(1-b \frac{t-t_{K}}{t_{A}-t_{K}}\right)$ with large values for the constant $b(b \simeq 10$ for $w=y=1$ and $b \simeq 5$ for $w=3, y=1)$. The reduction of the surface tension, shown in the inset of Fig. 2 as function of droplet radius $R=\frac{2 \sigma(t)}{T s_{c}(t)}$, is caused by the fact that $\mathrm{RSB}$ recognizes the existence of structures whose interface matches are less costly.

The driving force for the decay of the metastable frozen solid is entropic. As usual for a replica based theory, $S_{c}$ is only the mean value of the logarithm of the number of states. While fluctuations of the entropy density vanish for infinite systems, $\overline{\left(\delta s_{c}\right)^{2}}=c_{c} / V$, they become relevant in finite subsystems like small droplets. For example we find for the configurational specific heat capacity at $T_{K}: \quad c_{c}=\frac{2 q^{* 3}\left(t_{K}\right)}{3 T_{K}}$. Configurational entropy fluctuations, i.e. fluctuations of the driving force for nucleation, act similarly as do field fluctuations in the random field Ising model (RFIM). In the RFIM simple statistical arguments yield a reduction of the surface tension $\sigma(R)=\sigma_{0}\left(1-\right.$ const. $\left.R^{-3 / 2}\right)$. The effect is caused by fluctuations, corresponding to a wetting of the interface by states that make the matching between inside and outside the drop easier. $\sigma(R)$ computed this way behaves very similar to our numerical results presented above. In the context of the RFIM, Villain [27] proposed that one can consider such a $\sigma(R)$ as the first step of a renormalization group argument to the surface tension and obtained $\sigma \propto R^{-\frac{d-2}{2}}$ from the integration of the flow equation. For an RFOT this leads to a droplet radius $R^{*} \propto\left(t-t_{K}\right)^{-2 / d}$ and a Vogel-Fulcher law: $\tau=\tau_{0} \exp \left[D T_{K} /\left(T-T_{K}\right)\right]$ for the activated dynamics as advocated in Ref [3]. Our results strongly support this wetting picture in entropic droplets. For practical applications in liquids, where it is hard to approach $T_{K}$ very closely, the present first step of the full renormalization group calculation, may well be sufficient.

In summary we have shown that the transition states responsible for the slow activated dynamics in glassy systems are given by instantons with new replica symmetry breaking (RSB). This new RSB is due to a changed distribution of states at an interface between a frozen solid and a state which explores various metastable states. We note, the present treatment also allows naturally the inclusion of effects that will lead to barrier height fluctuations and non-exponential glassy relaxation.

We thank Jean-Philippe Bouchaud for helpful discussions. This research was supported by the Ames Laboratory, operated for the U.S. Department of Energy by Iowa State University under Contract No. W-7405-Eng82 (M.D. and J. S.), a Fellowship of the Institute for
Complex Adaptive Matter (M.D.), and the National Science Foundation grant CHE-0317017 (P. G. W.).

[1] T. R. Kirkpatrick and P. G. Wolynes, Phys. Rev. A 35, 3072 (1987).

[2] T. R. Kirkpatrick and P. G. Wolynes, Phys. Rev. B 36, 8552 (1987).

[3] T. R. Kirkpatrick and D. Thirumalai, and P. G. Wolynes, Phys. Rev. A 40, 1045 (1989).

[4] R. Monasson, Phys. Rev. Lett. 75, 2847 (1995).

[5] M. Mezard and G. Parisi, Phys. Rev. Lett. 82, 747 (1999).

[6] S. Franz and G. Parisi, J. Phys. I (France) 5, 1401 (1995).

[7] S. Franz and G. Parisi, Phys. Rev. Lett. 79, 2486 (1997).

[8] D. J. Gross, I. Kanter, and H. Sompolinsky, Phys. Rev. Lett. 55, 304 (1985).

[9] A. Crisanti and H.-J. Sommers, Z. Phys. B: Cond. Mat. 87, 341 (1992).

[10] T. R. Kirkpatrick and D. Thirumalai, Phys. Rev. Lett. 58, 2091 (1987).

[11] L. F. Cugliandolo, J. Kurchan, Phys. Rev. Lett. 71, 173 (1993).

[12] G. Biroli and J.-P. Bouchaud, Journ. of Chem. Phys., 121, 7347 (2004).

[13] X. Xia and P. G. Wolynes, Proc. Natl. Acad. Sci. 97, 2990 (2000);V. Lubchenko, P. G. Wolynes, Journ. of Chem. Phys. 121, 2852 (2004).

[14] X. Xia and P. G. Wolynes, Phys. Rev. Lett. 86, 5526 (2001).

[15] U. Tracht et. al., Phys. Rev. Lett. 81, 2727 (1998); E. V. Russel and N. E. Israeloff, Nature 408, 695 (2000); X. H. Qiu and M. D. Ediger, Jour. Phys. Chem. B 107, 459 (2003).

[16] V. Lubchenko, P. G. Wolynes, Phys. Rev. Lett. 87, 195901 (2001).

[17] S.Wu, H. Westfahl Jr., J. Schmalian, and P. G. Wolynes, Chem. Phys. Lett. 359, 1 (2002).

[18] J. Schmalian and P. G. Wolynes, Phys. Rev. Lett. 85, 836 (2000);H. Westfahl Jr., J. Schmalian, and P. G. Wolynes, Phys. Rev. B 64, 174203 (2001).

[19] G. Parisi, preprint, cond-mat/9411115

[20] S. Takada, P. G. Wolynes, J. Chem. Phys. 107, 9585 (1997).

[21] S. Franz, preprint cond-mat/0412383 Of the two approaches discussed, the quenched one yields a description for entropic droplets consistent with RFOT.

[22] G. Parisi, G. Ruocco, and F. Zamponi, Phys. Rev. E 69, 061505 (2004).

[23] A. Barrat, S. Franz, and G. Parisi, J. Phys. A: Math. Gen. 30, 5593 (1997).

[24] J. S. Langer, Annals of Physics 41, 108 (1967).

[25] C. Unger and W. Klein, Phys. Rev. B 28, 445 (1983).

[26] G. Biroli and J.-P. Bouchaud, Europhys. Lett. 67, 21 (2004).

[27] J. Villain, J. Physique 46, 1843 (1985). 